\section{PASE: Program for analysis of structural equations}

\author{
LEE M. WOLFLE \\ Virginia Polytechnic Institute and State University \\ Blacksburg, Virginia 24061
}

The program for analysis of structural equations (PASE) is a microcomputer program designed to provide the causal modeler with all of the usually desired estimates of coefficients in recursive causal models. The program is user friendly, in that it is interactive and selfdocumenting and requires only a series of option selections by the user. The program requires the input of a zero-order correlation matrix either from an existing file or from the keyboard. Output includes all usual regression coefficients, plus total causal effects decomposed into direct and indirect causal effects.

System Requirements. PASE was written for an Apple II or Apple II Plus microcomputer that utilizes APPLESOFT BASIC. System configuration must be a minimum of 48-KB RAM and one disk drive operating on DOS 3.3. The program provides support for, but does not require, a printer for hard-copy output.

Introduction to the Analysis of Structural Equations. One of the most important advances in social research methodology in the past 15 years has been the introduction (Duncan, 1966) of causal modeling techniques to the social sciences. On one hand, this development has been important to social theory, for the techniques of causal modeling provide an explicit link between theory and the equations used to test the hypothesized relationships. On the other hand, while the estimation methods of causal model coefficients is not new, the techniques have proved to be invaluable aids in the interpretation of social data. One of the most important of these interpretive aids in causal modeling is the decomposition of zeroorder associations among variables into various causal components (see Wolfle, 1980). Useful introductions to the analysis of structural equations can be found in Duncan (1975) and Kenny (1979).

Program Algorithm and Output. PASE is an appropriate tool for estimating hierarchical, recursive causal models. Because such models depend upon least squares estimation procedures, PASE provides all of the usual regression results and thus may be used as a multipleregression program. But in addition, PASE provides estimates of total causal effects and decomposes these into direct and indirect components (see Finney, 1972, for the interpretation of indirect causal effects, and Alwin \& Hauser, 1975, for a presentation of the general method for decomposing causal effects).

PASE permits the input of new correlation matrices along with means and standard deviations, if desired. All data matrices can be saved to disk in sequential form for future analyses. The program thus permits either the input of new matrices or the reading of previously saved data. Data matrices can be reviewed and corrected, truncated, or expanded to the maximum-sized matrix (17 variables) analyzable within the 48-KB memory limits of the compiled version of PASE.

Once the data have been input, reviewed if desired, changed if necessary, and saved to disk as recommended, the program prompts the user for the number of equations in the causal model. The program next asks the user to specify the dependent variable, followed by a list of the independent variables or causes of the variable specified as dependent. The program next requests the user to specify the causal order of variables. For example, the causal model shown in Figure 1 specifies that variables $\mathrm{X}(5), X(6)$, and $\mathrm{X}(7)$ occur in one causal block; $X(4)$ occurs by itself in a causal block, and $X(2)$ and $\mathrm{X}(3)$ occur together in a third causal block. With this information, the program proceeds with the calculation of all regression coefficients and then calculates all total causal effect coefficients, both standardized and metric, and decomposes these into direct and indirect causal components. (If one desires, the noncausal component of an association may be calculated by the simple expedient of subtracting the total causal effect from the zero-order association.)

The output of PASE has been organized for easy review. The output menu gives the user the option of reviewing the regression results, the regression ANOVA table, the R-squares among the independent variables, and the decomposition of causal effects. If desired, all of these results may be directed to a printer.

The regression results include all metric slopes, beta weights, standard errors, and $t$ ratios for the independent variables. Also included is the value of the intercept and the R-square for the regression.

The ANOVA table includes the regression sum of squares, the residual sum of squares, and the total sum of squares. The appropriate degrees of freedom are presented, along with the regression and residual mean squares and the $F$ ratio. The table also presents the standard error of estimate and restates the R-square.

The R-squares among the independent variables may be viewed. A high value among these may suggest the presence of multicollinearity, which, if present, causes regression coefficients to be unstable in the face of slight changes in sample size or zero-order correlations. In addition, standard errors are often inflated, and highly correlated independent variables often (and implausibly) have regression coefficients of opposite sign.

The table of causal decomposition presents the total effect of each independent variable, along with its direct effect and indirect causal effect. If there are no intervening variables between the causal independent variable 


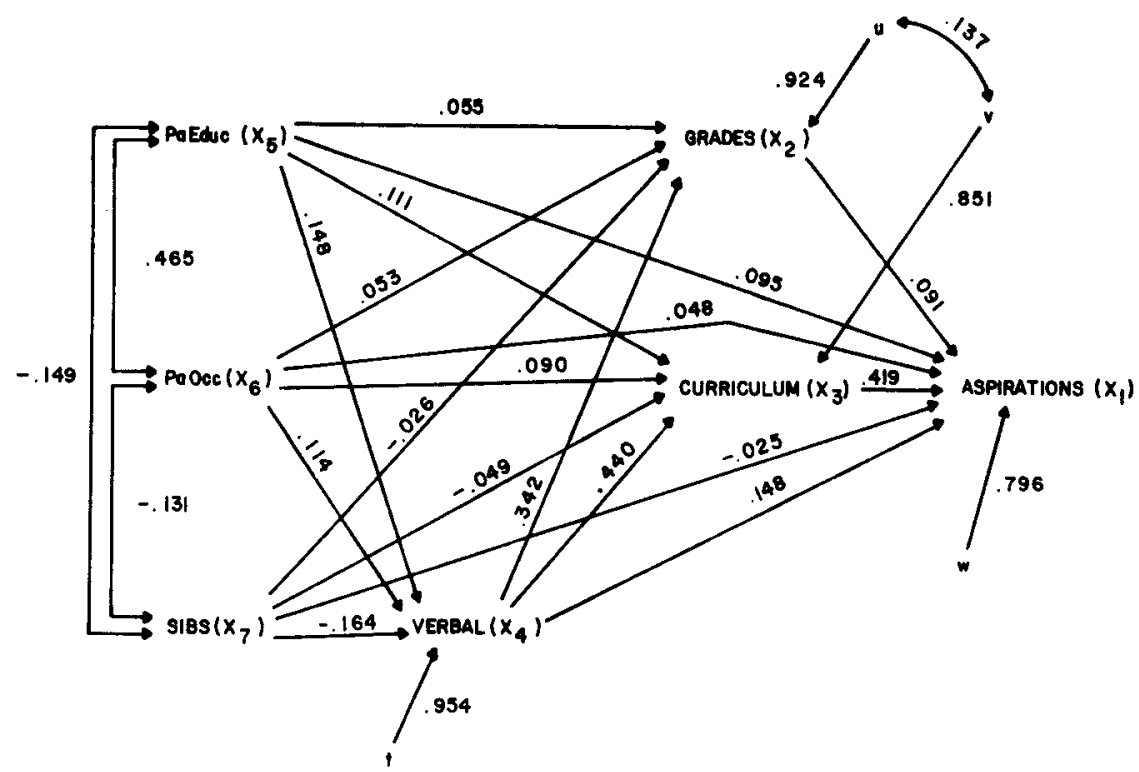

Figure 1. Block-recursive equation model of educational aspirations (Source: Heyns, 1974).

and the caused dependent variable, then the total effect is the direct effect. Table 1 shows the kind of output obtained from this portion of the PASE output routine. The coefficients pertain to the causal model shown in Figure 1. Only a brief perusal of Table 1 indicates the interpretive advantages of PASE, for it is clear that the social background variables $[X(5)=$ father's education, $X(6)=$ father's occupation, and $X(7)=$ number of siblings] manifest their causal impact on educational aspirations, $\mathrm{X}(1)$, predominantly indirectly and primarily through their influence on the placement of students in college preparatory academic tracks.

Program Accuracy. Users of PASE should have the opportunity to worry about the substance of their causal model (which is the most difficult part of causal modeling; Duncan, 1975) without having to worry about the accuracy of PASE's calculations. In order to check the numerical accuracy of PASE, Longley's (1967) data, as reported by Beaton, Rubin, and Barone (1976), were used to perform a six-variable multiple-regression analysis using both PASE and the NEW REGRESSION procedure in SPSS (Nie, Hull, Jenkins, Steinbrenner, \& Bent, 1975). Comparison of the SPSS and PASE metric

Table 1

Decomposition of Causal Effects (Standardized)

EFFECTS TO VARIABLE: 1

\begin{tabular}{lrrrr} 
FROM & TOTAL & & DIRECT & \multicolumn{1}{l}{ INDIRECT } \\
\cline { 5 - 5 } VAR. 5 & .2007 & & .0952 & .1056 \\
VAR. 6 & .1321 & & .0482 & .0840 \\
VAR. 7 & -.1078 & & -.0251 & -.0829 \\
VAR. 4 & .3634 & & .1476 & .2155 \\
VAR. 2 & .0910 & .0910 & 0 \\
VAR. 3 & .4192 & .4192 & 0
\end{tabular}

and standardized regression coefficients showed in every case that the coefficients differed by no more than \pm .0001 . Users of PASE may be assured that their numeric results are at least as accurate as regression results produced by SPSS on an IBM mainframe computer.

Computation Time Efficiency. Although the PASE disk includes the PASE source program, for most applications the user will want to use the compiled version. The compiled version of PASE is more than three times as fast as the source program. Several examples of approximate run times were calculated. The estimation of the coefficients for X(1) in Figure 1 took $14 \mathrm{sec}$. A model reported by Sewell and Hauser (1975), which included 13 independent variables in seven causal blocks, took $2.65 \mathrm{~min}$. With additional time spent inputting data, responding to program options, and examining the output, the actual user time would, of course, be longer.

Availability. PASE is available from the author, College of Education, Virginia Polytechnic Institute and State University, Blacksburg, Virginia 24061. Please enclose one blank 5.25 -in. soft-sectored floppy disk. A users' guide is also available; to cover printing costs, please enclose a check in the amount of $\$ 1.00$ made out to AES Division/College of Education.

\section{REFERENCES}

Alwin, D. F., \& Hauser, R. M. The decomposition of effects in path analysis. American Sociological Review, 1975, 40, $37-47$.

Beaton, A. E., Rubin, D. B., \& Barone, J. L. The acceptability of regression solutions: Another look at computational accuracy. Journal of the American Statistical Association, 1976, 71, 158-168.

Duncan, O. D. Path analysis: Sociological examples. American Journal of Sociology, 1966, 72, 1-16. 
Duncan, O. D. Introduction to structural equation models. New York: Academic Press, 1975.

Finney, J. M. Indirect effects in path analysis. Sociological Methods and Research, 1972, 1, 175-186.

HEYns, B. Social selection and stratification within schools. American Journal of Sociology, 1974, 79, 1434-1451.

Kenny, D. A. Correlation and causality. New York: Wiley, 1979.

LONGLEY, J. W. An appraisal of least squares programs for the electronic computer from the point of view of the user. Journal of the American Statistical Association, 1967, 62, 819-841.
Nie, N. H., Hull, C. H., Jenkins, J. G., Steinbaenner, K., \& BENT, D. H. Statistical package for the social sciences. New York: McGraw-Hill, 1975.

SEwell, W. H., \& HAUser, R. M. Education, occupation, and earnings: Achievement in the early career. New York: Academic Press, 1975.

Wolfle, L. M. Strategies of path analysis. American Educational Research Journal, 1980, 17, 183-209.

(Accepted for publication October 25, 1982.) 